\title{
Evaluation of engineered AAV capsids for hepatic factor IX gene transfer in murine and canine models
}

\author{
David M. Markusic ${ }^{{ }^{*}}\left(\mathbb{D}\right.$, Timothy C. Nichols ${ }^{2}$, Elizabeth P. Merricks², Brett Palaschak ${ }^{1}$, Irene Zolotukhin ${ }^{1}$, \\ Damien Marsic ${ }^{1}$, Sergei Zolotukhin ${ }^{1}$, Arun Srivastava ${ }^{1}$ and Roland W. Herzog ${ }^{1 *}$
}

\begin{abstract}
Background: Adeno-associated virus (AAV) gene therapy vectors have shown the best outcomes in human clinical studies for the treatment of genetic diseases such as hemophilia. However, these pivotal investigations have also identified several challenges. For example, high vector doses are often used for hepatic gene transfer, and cytotoxic T lymphocyte responses against viral capsid may occur. Therefore, achieving therapy at reduced vector doses and other strategies to reduce capsid antigen presentation are desirable.

Methods: We tested several engineered AAV capsids for factor IX (FIX) expression for the treatment of hemophilia B by hepatic gene transfer. These capsids lack potential phosphorylation or ubiquitination sites, or had been generated through molecular evolution.

Results: AAV2 capsids lacking either a single lysine residue or 3 tyrosine residues directed substantially higher coagulation FIX expression in mice compared to wild-type sequence or other mutations. In hemophilia B dogs, however, expression from the tyrosine-mutant vector was merely comparable to historical data on AAV2. Evolved AAV2-LiC capsid was highly efficient in hemophilia B mice but lacked efficacy in a hemophilia B dog.

Conclusions: Several alternative strategies for capsid modification improve the in vivo performance of AAV vectors in hepatic gene transfer for correction of hemophilia. However, capsid optimization solely in mouse liver may not predict efficacy in other species and thus is of limited translational utility.
\end{abstract}

Keywords: AAV, Gene therapy, Hemophilia B, Factor IX, Capsid

\section{Background}

Adeno-associated viral (AAV) vectors represent some of the most powerful tools for in vivo gene transfer to a variety of organs. For example, impressive successes with ocular gene transfer have been reported, resulting in reversal of inherited forms of blindness [1]. AAV vectors have also been in clinical development for hepatic gene transfer over the past decade, primarily to express coagulation factors in hepatocytes for the treatment of the $\mathrm{X}$-linked bleeding disorder hemophilia. Most recently,

\footnotetext{
*Correspondence: dmarkusic@ufl.edu; rherzog@ufl.edu

${ }^{1}$ Department of Pediatrics, University of Florida, Gainesville, FL 32610, USA

Full list of author information is available at the end of the article
}

curative levels of factor VIII (FVIII) and factor IX (FIX) were achieved in patients with hemophilia A or hemophilia $B$, respectively $[2,3]$. This was achieved using viral capsids derived from several different serotypes. However, in some cases very large vector doses were required. The viral capsid is a major determinant of AAV tropism, and some capsids have not performed as efficiently in humans as they did in preclinical studies. In particular, transduction of mouse liver has been more effective than human liver. Thus, the search for optimal serotypes/capsids for transduction of human hepatocytes continues, for instance through studies in non-human primates or in "humanized" mice with chimeric mouse-human livers $[4,5]$. Another obstacle that continues to negatively affect gene therapy for hemophilia is the activation of 
capsid-specific $\mathrm{CD}^{+} \mathrm{T}$ cells [6]. Hepatocytes cross-present the viral input capsid antigen via MHC I, following endosomal escape of the infecting viral particles, ubiquitination, and proteasomal degradation [7-9]. Capsid-specific cytotoxic T lymphocytes can target AAV transduced hepatocytes, resulting in loss of expression and toxicity $[10,11]$. Immune suppression regimens have been developed to counter these $T$ cell responses $[12,13]$.

Enhancing the performance of AAV gene transfer could potentially result in therapy at lower vector doses, which should also reduce capsid antigen presentation. It is hoped that reducing capsid antigen below a threshold level will resolve this issue. We sought to develop vectors that are effective at lower vector doses and postulated that AAV capsids could be engineered to alter intracellular trafficking post-entry of the vector into target cells, thereby enhancing delivery to the nucleus. Our studies of the life cycle of AAV2 demonstrated that shunting of viral particles to the proteasome and subsequent degradation is promoted by phosphorylation of capsid tyrosine (Y) residues, which provide a signal for capsid polyubiquitination [14]. Therefore, we hypothesized that substitution of surfaced exposed tyrosine residues in the AAV2 capsid would significantly reduce proteasomal processing of capsid. Thus, we individually mutated seven surface exposed tyrosine residues at positions (252, 272, 444, $500,700,704$, and 730) to phenylalanine and observed enhanced in vitro transduction at residues 444,500 , and 730 , that was not augmented by either tyrosine kinase or proteasome inhibition. These mutant capsids showed a reduction in polyubiquitination and directed increased trafficking to the nucleus [15]. The combination of Y444F, Y500F, and Y730F mutations (AAV2-(Y-F)-M3) resulted in the best improvement in vitro, and in vivo for murine hepatocyte transduction [16]. As immunological presentation of capsid antigen is dependent on proteasome degradation, the M3 mutation protected AAV transduced human hepatocytes in vitro and murine hepatocytes in vivo from $\mathrm{CD}^{+} \mathrm{T}$ cell targeting and killing, thus validating our hypothesis [9].

Besides surface-exposed tyrosine residues, serine and threonine residues represent potential phosphorylation sites on the AAV capsid. Elimination of lysine residues may directly prevent ubiquitination, as opposed to the indirect method of preventing capsid phosphorylation, which serves as a signal for ubiquitination [17]. After identifying an optimal combination of Y-F variants for hepatocyte gene transfer, we therefore sought to determine if substitution of alternative kinase targets serine (S) and threonine $(\mathrm{T})$ and ubiquitin target lysine $(\mathrm{K})$ would further improve transduction $[18,19]$. In an alternative strategy, we developed a combinatorial library, initially based on the AAV2 cap sequence, with an unprecedented complexity to select for capsid variants that optimally infect hepatocytes [20, 21]. As proof-of-principle, we selected for two variants, termed AAV2-LiA and AAV2$\mathrm{LiC}$, with high tropism for murine liver [20]. Here, we evaluated AAV2-LiA and AAV2-LiC variants for correction of murine hemophilia $B$ by hepatic gene transfer. Further we compared several engineered AAV2 and AAV8 capsid variants with $\mathrm{Y}, \mathrm{S}, \mathrm{T}$, and $\mathrm{L}$ substitutions for in vivo $F 9$ gene transfer to mouse liver. Finally, AAV2(Y-F)-M3 and AAV2-LiC were tested in a large animal model, hemophilia B dogs, for FIX expression following liver-directed gene transfer.

\section{Methods}

\section{$A A V$ vector construction and production}

Site-directed mutagenesis and plasmids pACGr2c2 and pACGr2c8 was performed as previously described using a two-stage PCR reaction $[15,18,19]$. AAV2-LiA and AAV2-LiC were identified by sequencing DNA isolated from liver tissue following three successive rounds of in vivo capsid library infection as previously described by Marsic et al. [20]. AAV vectors expressing human F9 from the liver-specific ApoE enhancer and $\alpha_{1}$-antitrypsin promoter were packaged into AAV2, AAV2-LiA, AAV2LiC, AAV2 variants (Table 1), AAV8, and AAV8 variants (Table 1) capsids as previously published [16].

\section{Experimental animals}

Murine studies were approved under University of Florida Institutional Animal Care and Use Committee protocol \#201503182. Male hemophilia B mice on a BALB/c background $\left(\mathrm{BALB} / \mathrm{c}-\mathrm{F}^{-/ \mathrm{Y}}\right)$ are bred onsite and have a targeted deletion of the murine $F 9$ gene [22, 23]. Male C57BL/6 mice were purchased from The Jackson Laboratory (Bel Harbor, ME). All studies were conducted in mice $6-8$ weeks old. Vector was infused by tail injection at either $1 \times 10^{10}$ or $1 \times 10^{11} \mathrm{vg}$ as indicated. When indicated, $\mathrm{BALB} / \mathrm{c}-\mathrm{F9}^{-/ \mathrm{Y}}$ mice received a subcutaneous immunization of 1 IU recombinant FIX protein in adjuvant (Sigma Adjuvant System S6322). Blood samples for $\mathrm{BALB} / \mathrm{c}-\mathrm{F} 9^{-/ \mathrm{Y}}$ mice were collected by tail transection into $3.8 \%$ citrate buffer $(0.38 \%$ final). For all C57BL $/ 6$ studies mice were bled from the retro-orbital plexus into heparinized micro-capillary tubes.

A canine hemophilia B dog colony is maintained at the Francis Owen Blood Research Laboratory (University of North Carolina at Chapel Hill). These animals have a missense mutation (E379G) in canine factor IX that results in misfolded cFIX protein and complete absence of cFIX protein in circulation. All experiments were conducted in male hemophilia $B$ dogs that were pre-screened for anti-AAV neutralizing antibodies prior to enrollment. All animals were housed in US Department of Agriculture 
Table 1 Summary of AAV variants and mouse strains tested

\begin{tabular}{|c|c|c|c|c|}
\hline Serotype & (Y-F) & $(T-V)$ & (K-E) & Mouse \\
\hline AAV2-(Y-F)-M3 & $444,500,730$ & & & C57BL/6 \\
\hline AAV2-(Y-F)-M3-T491V & $444,500,730$ & 491 & & C57BL/6 \\
\hline AAV2-(Y-F)-M3-(T491+550V) & $444,500,730$ & 491 and 550 & & C57BL/6 \\
\hline AAV2-K544E & & & 544 & C57BL/6 \\
\hline AAV2-K556E & & & 556 & C57BL/6 \\
\hline AAV2-LiA & 444 and 500 & & & $\mathrm{BALB} / \mathrm{C}-\mathrm{HB}$ \\
\hline AAV2-LiC & 500 & & & $\mathrm{BALB} / \mathrm{C}-\mathrm{HB}$ \\
\hline AAV8 & & & & C57BL/6 \\
\hline AAV8-T494V & & 494 & & C57BL/6 \\
\hline AAV8-(Y275+447+733F) & 275,447 , and 733 & & & C57BL/6 \\
\hline
\end{tabular}

approved facilities and the experimental protocol was approved by the Institutional Animal Care and Use Committee at UNC-Chapel Hill. The weights and ages of animals at the time of vector infusion are listed in Table 2. Plasma was collected per study protocol and shipped to UF. CBC, WBCT, and other routine blood tests were performed onsite at UNC-Chapel Hill on a scil Vet abc cell counter calibrated for canine cells. Vector was infused by portal vein (AAV2-(Y-F)-M3) and saphenous vein (AAV2$\mathrm{LiC})$ at the indicated vector doses as published [24].

\section{Blood/plasma analysis or FIX, coagulation, and antibody assays}

Plasma samples from hemophilia B mice and dogs were analyzed using a modified activated partial thromboplastin time assay (aPTT). The \%hFIX activity in mice or \%cFIX activity in dogs was determined from a loglog standard curve generated from dilutions of normal human and dog plasma. Inhibitory antibodies to hFIX or cFIX were measured by Bethesda assay as previously described [25, 26]. One Bethesda unit is defined as the reciprocal of the dilution of test plasma at which $50 \%$ of cFIX or hFIX activity is inhibited. Measurements were carried out on a Diagnostica Stago STart Hemostasis Analyzer (Parsippany, NJ). Levels of hFIX antigen in mice and IgG1 antibodies to FIX were measured by enzymelinked immunosorbent assay (ELISA) as previously described [25]. Levels of cFIX antigen in mouse plasma were measured by ELISA (Affinity Biologicals CFIX-EIA) using a standard derived from normal pooled canine plasma following the manufacturer's protocol.

\section{Detection of AAV NAB}

Neutralizing antibodies (NABs) against AAV2(Y-F)-M3 or AAV2-LiC vector particles were measured by inhibition of in vitro GFP transduction of HeLa cells and reported as the reciprocal dilution of plasma that resulted in $50 \%$ inhibition of transduction relative to control cells transduced with virus alone. Cells were co-infected with adenovirus to enhance AAV mediated GFP expression.

\section{Statistical analysis}

Results are presented as mean \pm SD. Differences between experimental groups were evaluated using one-way ANOVA with Turkey multiple comparisons test or unpaired $\mathrm{T}$ tests when only two groups were compared.

\section{Results}

Lysine mutant capsid as potential alternative to tyrosine mutations for improved factor IX expression

Previous reports have shown that elimination of surface exposed lysine residues, a putative site for polyubiquitination, on AAV2 leads to an increase in murine hepatocyte transduction efficiency over wild-type capsid $[19,27]$. Yet a limitation of these studies was the use of a non-secreted transgene product, GFP or luciferase, that hindered quantitative comparison of transgene expression and did not account for systemic delivery as needed in the treatment of hemophilia. Secondly, some of these studies were conducted in reference to wild-type AAV2 and lack direct comparison to the optimized AAV2(YF)-M3 capsid [16]. In the present study, we evaluate the efficacy of two single (K-E) substitutions at positions

Table 2 Overview of hemophilia B canine studies

\begin{tabular}{|c|c|c|c|c|c|c|}
\hline Dog & Serotype & Route & Dose (vg/kg) & Weight (kg) & Age (year month) & Gender \\
\hline 019 & AAV2(Y-F)-M3 & PV & $2 \times 10^{11}$ & 27.8 & 2 year 1 month & Male \\
\hline O58 & AAV2(Y-F)-M3 & PV & $6 \times 10^{11}$ & 23.2 & 2 year 6 month & Male \\
\hline Bruce & AAV2-LiC & IV & $6 \times 10^{11}$ & 21.4 & 4 year 1 month & Male \\
\hline
\end{tabular}


544 or 556 compared against both AAV2 and AAV2(YF)-M3 vectors in C57BL/6 mice using a ssAAV-ApoEhAAT- $h F 9$ vector (expression from the ApoE hepatocyte control region and enhancer, combined with human $\alpha_{1}$ antitrypsin promoter) that expresses human factor IX protein (hFIX) from a liver specific promoter summarized in Table 1 . Although a $\mathrm{K}$ to $\mathrm{R}$ substitution conserves the amino acid charge, in our hands we were unable to generate suitably infectious viral particles $[19,27]$.

Mice were injected in the tail vein with $1 \times 10^{10} \mathrm{vg}$ of each vector, and hFIX levels were determined in plasma by ELISA at 1 month after gene transfer. On average, AAV2-K544E yielded a 3.6-fold increase in systemic hFIX expression compared to AAV2, although not reaching a statistically significant difference. In contrast, AAV2K556E treated mice had a significant and more robust increase in levels of circulating hFIX protein compared to AAV2 (13.5-fold, Fig. 1a). These did not quite reach the levels of AAV2(Y-F)-M3 treated mice, which expressed 2.3-fold higher hFIX as compared to AAV2-K556E transduced mice. Hence, K556 may be a critical site for AAV2 capsid ubiquitination, and elimination of this single lysine residue increases transgene expression to near the levels achieved with optimal multiple tyrosine mutant capsid.

Next, we asked if a single (T491V) or double (T491V and $\mathrm{T} 550 \mathrm{~V}$ ) threonine mutants combined with AAV2(Y-F)-M3 would further enhance murine hepatocyte gene transfer, as has been suggested in prior work [18]. In contrast to those earlier data obtained with non-secreted reporter genes, we did not observe an improvement in hFIX levels with the addition of either $\mathrm{T}$ mutation (Fig. 1a). AAV2-(Y-F)-M3 injected mice expressed 31.5fold more hFIX compared to wild-type AAV2, consistent with our previously published findings (Fig. 1a) [16].

\section{Inability to further improve AAV8 performance in murine liver}

Among naturally occurring serotypes, AAV8 has the highest transduction efficiency of murine liver. We have recently shown that substitution of surface exposed lysine $(\mathrm{K})$ residues in AAV8 did not lead to a substantial improvement in murine liver transduction and in some instances, was detrimental [19]. Therefore, we tested if instead, substitution of surfaced exposed tyrosine $(\mathrm{Y})$

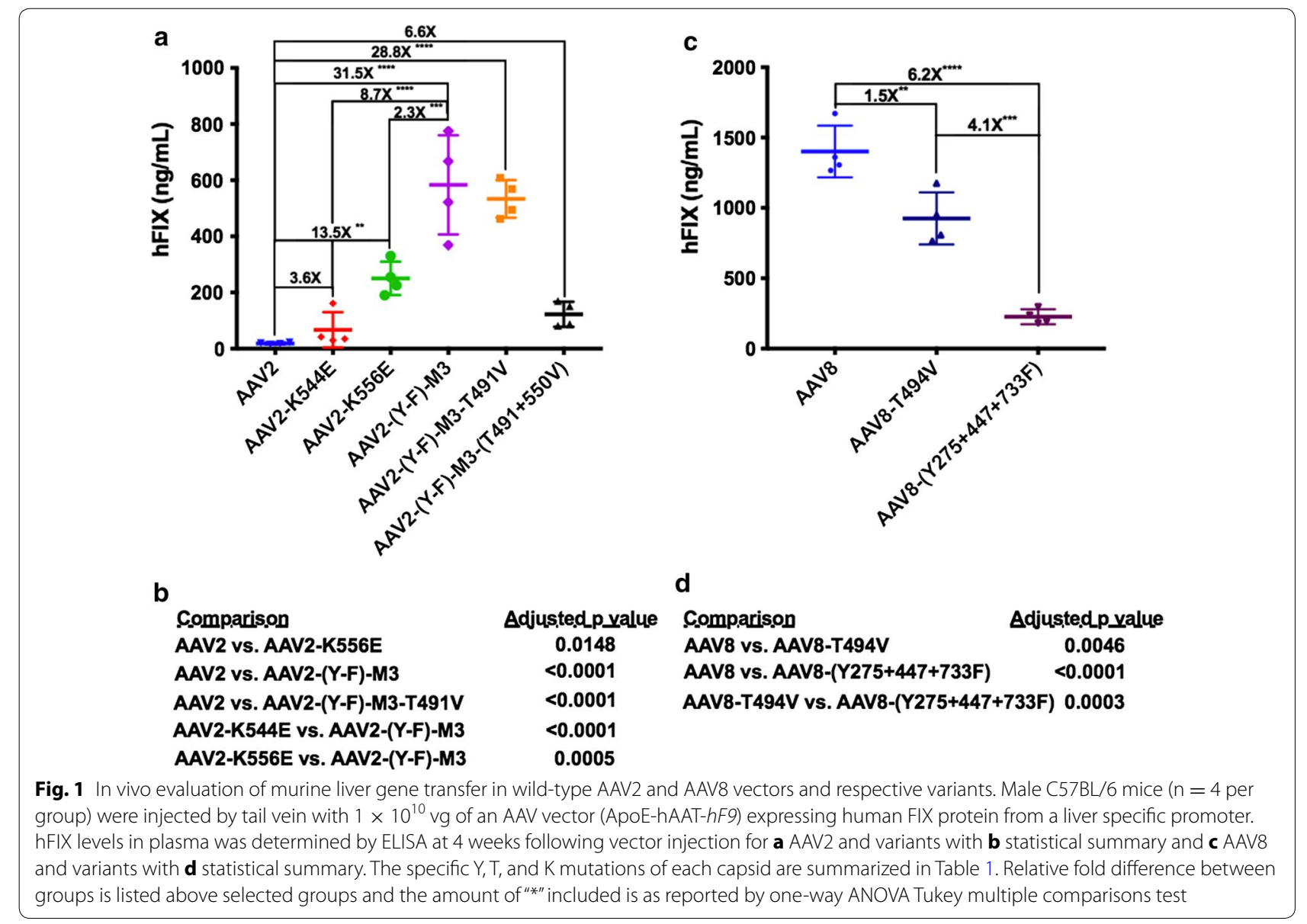


or threonine $(\mathrm{T})$ residues could further improve hepatic AAV8 gene transfer in mice. AAV2 and AAV8 amino acid alignment and surface residue prediction resulted in the identification of AAV8 Y residues 275, 447, and 733 and a $\mathrm{T}$ residue at position 494, which maps closely to those identified in AAV2, excluding Y-275. However, neither AAV8(Y-F)-M3 nor AAV8-T494V vectors improved hFIX expression levels compared to wild-type AAV8 (Fig. 1b), similar to our previous findings with lysine substitutions.

\section{Partial correction of canine hemophilia B with AAV2-(Y-F)-M3 vector}

An advantage in development of novel therapies for hemophilia is the existence of a well characterized large animal model, in form for hemophilic dogs $[28,29]$. Of the various $\mathrm{Y}, \mathrm{K}, \mathrm{S}$, and $\mathrm{T}$ mutation capsids, AAV2-(Y-F)M3 showed the strongest improvement in hFIX expression in mouse liver compared to the parent capsid, in this case AAV2. Previously, several hemophilia B dogs injected in the mesenteric or portal vein with an AAV2(ApoE) $)_{4}$-hAAT-cF9 vector, expressing canine FIX (cFIX), at doses of approximately $1 \times 10^{12} \mathrm{vg} / \mathrm{kg}$ achieved sustained therapeutic levels of $\sim 5 \%$ of normal for multiple years [30,31]. Based on these studies, we elected to test AAV2-(Y-F)-M3 in the canine model. We constructed a vector that expresses cFIX from the ApoE/hAAT combination used in the mouse experiments described above (thus similar to the hAAT promoter combined with 4 copies of the ApoE enhancer in the previously published studies). The vector was to be administered to male hemophilia B dogs from the UNC-Chapel Hill colony. These dogs have a missense mutation, leading to expression of cFIX in hepatocytes but lacking circulating cFIX antigen and activity [32]. All prospective subjects were screened for lack of AAV capsid neutralizing antibodies using an in vitro neutralizing antibody (NAB) assay. Since in mice we observed a significant enhancement in hFIX expression following portal vein delivery of AAV(YF) mutant vector compared to peripheral vein injection [15], we chose to administer the AAV2-(Y-F)-M3 vector via the portal vein.

Two male dogs O19 2.1 years and O58 2.5 years were chosen, see Table 2 for details. The first animal, O19, was injected at a vector dose of $2 \times 10^{11} \mathrm{vg} / \mathrm{kg}$, approximately fivefold lower than historically treated AAV2 dogs, into the portal vein [24]. To cover the animal during the surgical intervention, O19 received coverage with normal dog plasma with a total of $450 \mathrm{~mL}$ on the day of injection followed by $300 \mathrm{~mL}$ on days 1,2 , and 3 post-injection. A hematoma was noted at the injection site on day 15 postinjection, and $150 \mathrm{~mL}$ plasma was administered on days $15,16,17$, and 18 . As a result of gene transfer, WBCT fell from pre-treatment of greater than 60 min to a mean of $21.8 \pm 2.2 \mathrm{~min}$ (calculated from day 29 onward) post gene therapy (Fig. 2a, blue tracing). Canine FIX activity, measured over a period of 97 days post treatment, ranged from 0.3 to $1.5 \%$ of normal. At study endpoint, cFIX activity was approximately $0.5 \%$ of normal (Fig. 2b, blue tracing). Clinical chemistry and $\mathrm{CBC}$ revealed a moderate reduction in platelet counts over the course of the study and a mild to moderate elevation in ALT prior to treatment and towards the end of the study on days 97 , 140, and 175 (Additional file 1: Table S1).

A second animal, O58, was injected with a threefold higher vector dose of $6 \times 10^{11} \mathrm{vg} / \mathrm{kg}$ and was followed over time. Dog O58 received $800 \mathrm{~mL}$ normal plasma over the course of the day of injection, followed by $350 \mathrm{~mL}$ on day $1,400 \mathrm{~mL}$ day 2 , and $200 \mathrm{~mL}$ on days 3,4 , and 5. There were no major complications or bleeds following vector injection. Clinical chemistry and $\mathrm{CBC}$ counts revealed a mild reduction in platelets and a slight elevation in hematocrit and hemoglobin (Additional file 2: Table S2). At this higher vector dose, WBCTs decreased from a pre-treatment value of $48 \mathrm{~min}$ to an average of $19.1 \pm 2.6 \mathrm{~min}$ (Fig. 2a, red tracing). A cFIX activity of around $1 \%$ of normal was achieved (Fig. 2b, red tracing), when the WBCT was 14-18 min. As expected for hepatic gene transfer to these animals with a $F 9$ missense mutation, gene transfer did not induce an inhibitor against cFIX in either dog (Additional file 3: Figure S1).

\section{Treatment of murine and canine hemophilia B using capsids evolved to optimally transduce murine liver}

Next, we investigated the efficacy of two AAV2 variants that were selected for murine hepatocyte tropism through repetitive rounds of screening a rationally designed capsid library [20]. AAV2-LiA contains two Y-F substitutions at 444 and 500 in addition to 12 additional mutations, while AAV2-LiC has only a single Y-F substitution at position 500 and 3 additional mutations (Table 1) [20]. Unlike AAV2-(Y-F)-M3, both AAV2-LiA and AAV2-LiC serotyped vectors are equivalent to AAV8 for hFIX expression in mouse liver, providing comparable levels following intravenous delivery. Therefore, we tested the efficacy of both AAV2-LiA and AAV2-LiC in hemophilia B mice (BALB/c- $F 9^{-/ Y}$ ) injected at a dose of $1 \times 10^{11} \mathrm{vg}$ with ssAAV-ApoE-hAAT- $h F 9$ vector. Mice were followed over time for hFIX protein levels by ELISA, and activity levels were derived from a standard curve in an aPTT assay. Mice treated with either vector expressed comparable levels of hFIX protein and had similar high activity, which reached normal to super physiological levels of up to $\sim 150 \%$ (Fig. 3a, b). Nine weeks following vector injection, vector treated and naïve mice were immunologically challenged with $1 \mathrm{IU}$ of recombinant 

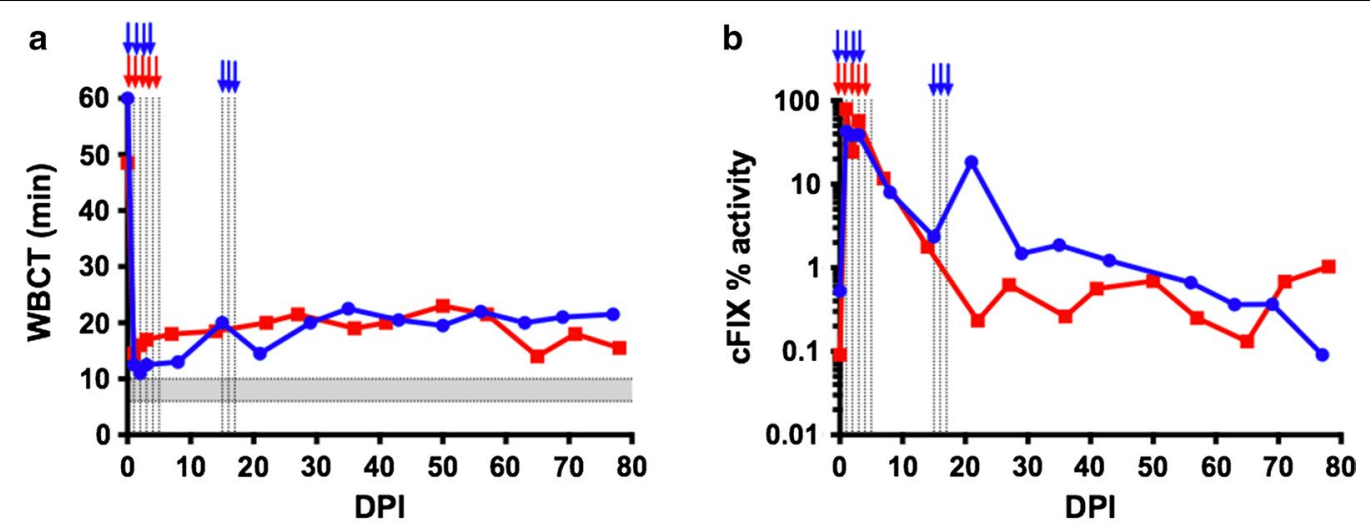

019 (AAV2-M3) PV 2x1011 vg/kg $\downarrow$ wt plasma O58 (AAV2-M3) PV 6x10 11 vg/kg $\downarrow$ wt plasma

Fig. 2 Hemophilia B dogs injected with two different doses of AAV2-(Y-F)-M3-ApoE-hAAT-cF9 vector do not express therapeutic levels of cFIX protein. a Whole blood clotting times (WBTC) and $\mathbf{b}$ CFIX activity of dogs $\mathrm{O} 19$ (blue $2 \times 10^{11} \mathrm{vg} / \mathrm{kg}$ ) and O58 (red $6 \times 10^{11} \mathrm{vg} / \mathrm{kg}$ ) over time. Colored arrows indicate infusion of wild-type dog plasma. Normal WBCT is indicated by shaded gray area on graph

hFIX protein in adjuvant. None of the animals treated with gene therapy developed antibodies against hFIX or had a reduction in circulating hFIX protein or coagulation activity (Fig. $3 \mathrm{a}-\mathrm{c}$ ), while naïve challenged BALB/ $\mathrm{c}-\mathrm{F} 9^{-/ \mathrm{Y}}$ mice developed robust anti-FIX titers. Thus, hepatic gene transfer had induced humoral tolerance to hFIX.

To test one of these capsids, which were highly efficient in hemophilic mice, in the hemophilia B dog model, we produced AAV2-LiC-ApoE-hAAT-cF9 vector. We confirmed in mice that the vector directed high levels of cFIX expression ( 30-fold higher compared to the AAV2-(Y-F)-M3 vector when normalized to vector dose, Fig. 4a). Three prospective animals were screened for the presence of pre-existing NAB against AAV2-LiC, and all three were negative. Based on previous outcomes with AAV8 vectors in hemophilia B dogs [33], and comparable gene transfer efficiency of AAV2-LiC with AAV8 in mice [20], we selected a vector dose of $6 \times 10^{11} \mathrm{vg} /$ $\mathrm{kg}$ with intravenous delivery into our subject (hemophilia B dog Bruce, 4.1 years, Table 2). Clinical chemistry and CBCs did not reveal any abnormalities over the course of the study, results are presented in Additional file 4: Table S3. However, Bruce achieved only a modest decrease in WBCT from $>60 \mathrm{~min}$ to an average of $34.4 \pm 3.8 \mathrm{~min}$ (Fig. 4b), without evidence for inhibitor formation (Additional file 3: Figure S1). As expected from the WBCTs, cFIX activity was consistently $<0.5 \%$ of normal (Fig. 4c). Thus, the high efficiency of FIX expression in hepatic gene transfer from peripheral vein in mice did not translate into efficacy in hemophilic dogs.

\section{Discussion}

Current gene therapy trials for the treatment of hemophilia A and B are all based on liver-directed gene transfer with AAV vectors injected into a peripheral vein [2, 3]. Different serotypes with liver tropism are used to transduce hepatocytes. Major advantages of liver gene transfer include the capacity of hepatocytes to efficiently secrete proteins into the circulation, their ability to perform the posttranslational modifications that are needed to produce biologically active FVIII and FIX, and induction of immune tolerance to the hepatocyte-expressed transgene product [34]. Current obstacles are the high vector doses that are typically required and activation of capsid-specific $\mathrm{CD}^{+} \mathrm{T}$ cells. Both problems can potentially be addressed by engineering the capsid. Informed by an improved understanding of the biology of the viral interactions with target cells, specific mutations can be designed to eliminate amino acids that promote proteasomal degradation of capsid, thereby limiting efficacy and increasing MHC I antigen presentation [17].

\section{Empirical evaluation of candidate mutations reveals alternative classes of amino acid changes that improve performance of different serotype capsids}

With the goal of eliminating either capsid phosphorylation or ubiquitination, we tested a variety of amino acid changes. Combinations of these may have synergistic effects. As of now, there is no method to predict their effects. Thus, these candidate mutations have to be empirically evaluated. Our initial focus was on Y-F mutations, which were most optimal for AAV2 in a combination of 3 specific capsid residues. In the case of AAV3, a 

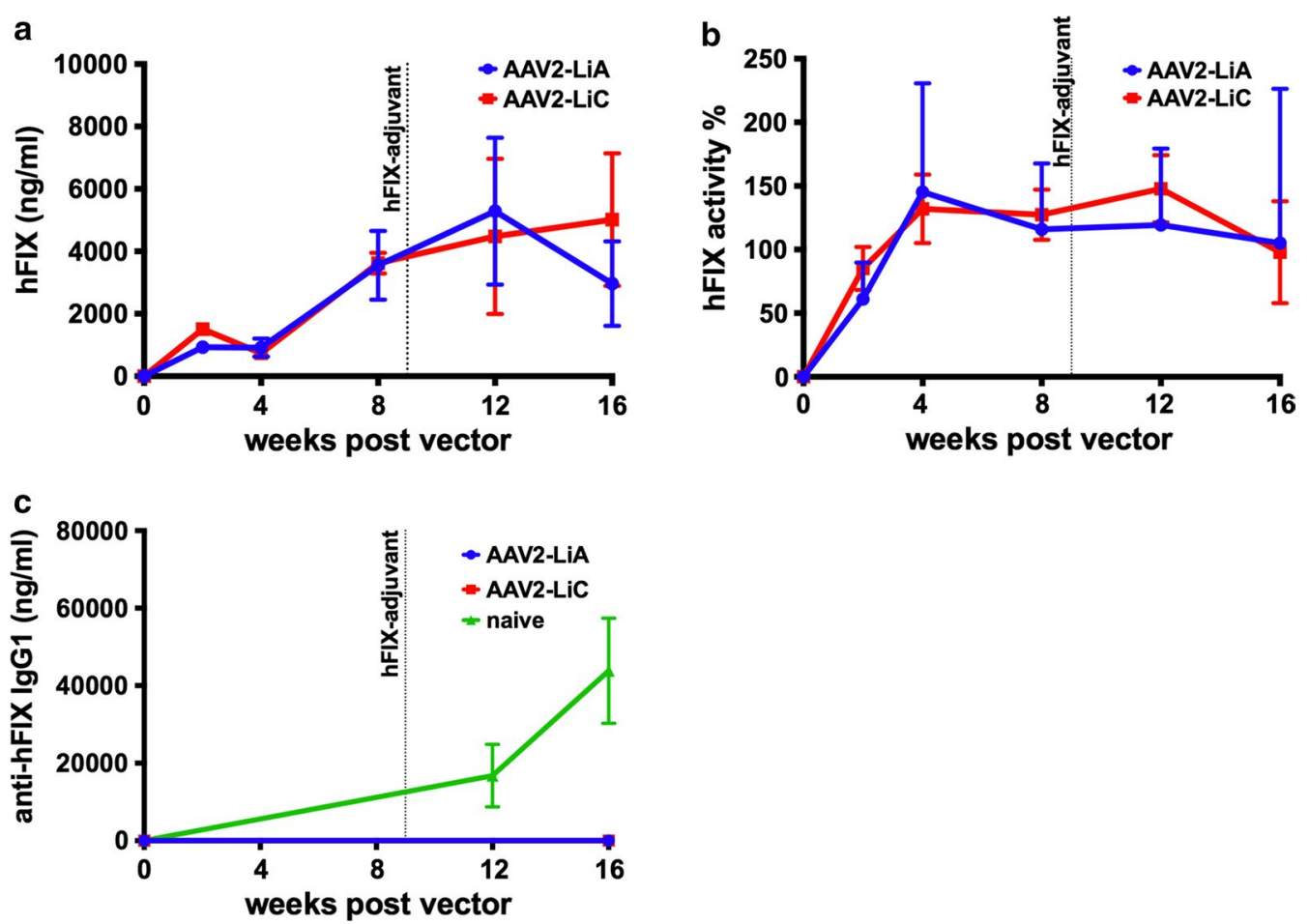

Fig. 3 AAV2-LiC capsid selected for murine hepatocyte tropism normalizes coagulation and induces tolerance in hemophilia B mice. Hemophilia B mice (BALB/C-F9- ${ }^{-N}$ ) were intravenously injected with $1 \times 10^{11} \mathrm{vg}$ of either AAV2-LiA or AAV2-LiC ApoE-hAAT-hF9 vector and followed over time for $\mathbf{a}$ hFIX protein levels $\mathbf{b} \%$ FIX coagulation activity, and $\mathbf{c}$ anti-hFIX IgG1 antibody titers. Horizontal dotted line in a-c denotes time of adjuvant-hFIX challenge in mice

serotype that infects human and primate hepatocytes but is ineffective for mouse, Y-F mutations only have a modest effect, while a combination of $\mathrm{S}-\mathrm{V}$ and $\mathrm{T}-\mathrm{V}$ mutations is more optimal [4, 35-37]. Recently, we began to evaluate K-E mutations [19]. Consistent with our published findings using reporter genes, K556E mutation substantially improved FIX expression from an AAV2 vector in hepatic gene transfer. Although not quite as effective as the optimal Y-F triple mutation, it is remarkable that elimination of a single lysine residue can improve efficacy by $\sim 1 \log$ (Figs. 1a, 4a). Unfortunately, it does not appear that this mutation can be combined with others without losing vector yields or infectivity, as changes in capsid structure may impact assembly [19]. More research is required to test whether the $\mathrm{K} 556 \mathrm{E}$ mutation indeed reduces AAV2 capsid ubiquitination and to determine the effect on MHC I presentation of capsid antigen.

Previously, we found that the addition of T491V to Y444,500,730F further improved transduction and transgene expression. However, the effect was more modest than the improvement observed for Y444,500,730F compared to single Y-F mutations. Compared to wild-type AAV2, most of the increase in efficacy can be attributed to the Y730F mutation. With each further optimization, improvement becomes more incremental. Although addition of T491V may somewhat further increase translocation of Y444,500,730F vector to the nucleus, this results in no further improvement in systemic FIX expression. The exact mechanism of enhanced gene transfer by specific combination of mutations is unknown. One potential explanation for the improvement in gene transfer with (Y444,500,730F) over the individual Y-F mutations is that the respective localization of these three residues may regulate ubiquitination based on integrative signaling [38].

\section{Engineering fails to improve the gold standard for mice, AAV8}

Next we investigated if engineering could improve murine hepatocyte transduction efficiency of AAV8. Using synonymous $\mathrm{Y}$ and $\mathrm{T}$ residues in AAV8 we generated two AAV8 variants AAV8-(Y-F)-M3 (275, 447, 733) and AAV8-T494V, which closely mimic previous AAV2 variants. As seen with AAV8K variants [19], both variants failed to improve hFIX expression over wild-type AAV8. AAV8 serotype vectors have exceptional natural tropism for murine hepatocytes in vivo [39]. Unlike AAV2, transduction efficiency is independent of delivery route [40], and upon cellular entry AAV8 viral particles 

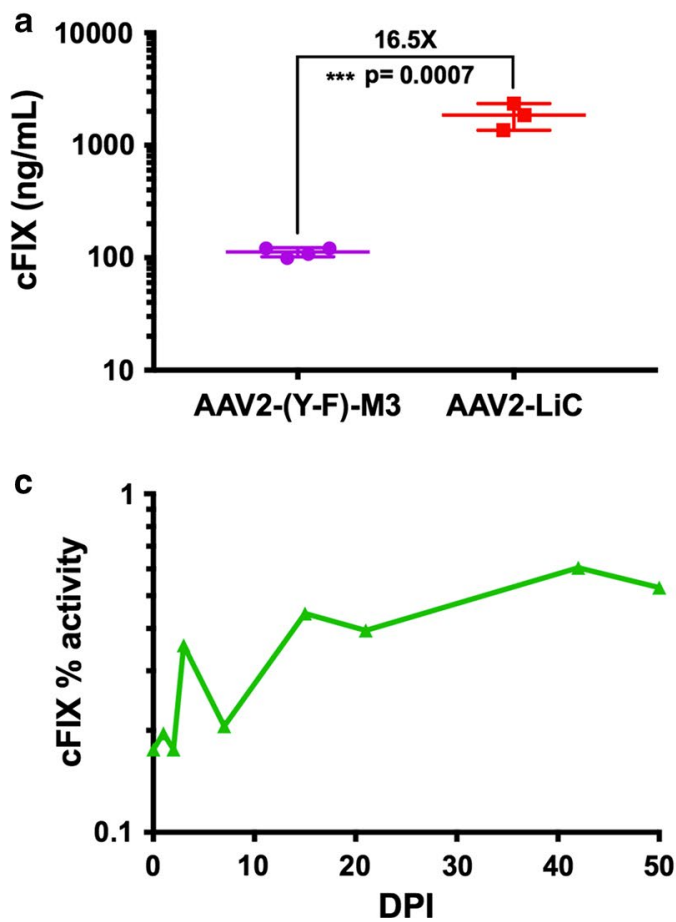

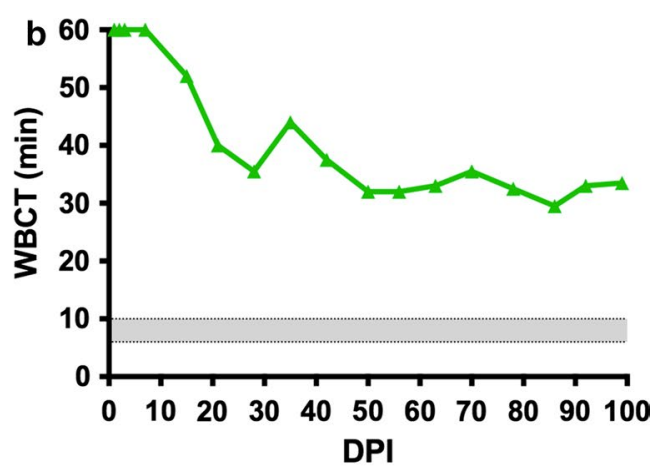

F. 4 Administration of AAV2-LiC-ApoE-hat-CF9 to a hemophilia B dog leads to only minimal correction of coagulation. a C57BL/6 mice were of circulating CFIX protein were measured by ELISA at 4 weeks post gene delivery. b, c A hemophilia B dog, Bruce, was peripherally injected with $6 \times 10^{11} \mathrm{vg} / \mathrm{kg}$ of an AAV2-LiC-ApoE-hAAT-CF9 vector and followed over time for changes in $\mathbf{b}$ WBCT and $\mathbf{c} \%$ CFIX activity. Fold difference between groups in $\mathbf{a}$ are listed above and the amount of "**" and $p$ value is included as reported by an unpaired T test

rapidly release their genetic cargo [39]. In contrast, even though AAV2 serotype vectors have been shown to infect nearly all murine hepatocytes, transgene expression is often restricted to a small fraction of hepatocytes [41]. We have shown that this is mediated in part by phosphorylation of the FKBP52 protein and suppression of second-strand synthesis [42, 43]. The kinetics of AAV8 vector uncoating, second strand synthesis, and transgene expression may explain how SsAAV8 vectors bypass this suppression. Our collective data suggest that in mice the ubiquitination-proteasome pathway is not rate limiting for AAV8 gene transfer in mouse liver. This is independently supported by studies that show proteasome inhibitors fail to enhance transgene expression following AAV8 gene transfer $[44,45]$, although this has been suggested to be dependent on the size of the transgene [45].

\section{No indication of toxicity from vector infusions in hemophilia B dogs}

Of the three dogs injected, only one animal O19 displayed a mild rise in alanine transaminase (ALT) levels, slightly above the upper limit of the reference range provided by the clinical diagnostic laboratory Antech Inc. This elevation was noted prior to vector infusion at baseline and remained in the normal range up to 3 weeks following vector infusion. ALT levels again rose above normal range on day 97 and remained so until the end of the study. Dog O58, which received a threefold higher vector dose compared to O19, maintained normal ALT levels suggesting that this mild elevation in ALT was unrelated to the vector. All animals had a mild reduction in platelet counts during follow-up. Perioperative reductions in platelet counts are probably due to fluid and normal canine plasma administration as well as some consumption at surgical wound sites. Even though some of the values are below the lower end of the reference range, they are not at a level that would be associated with clinical bleeding. Dogs O19 and O58 had mild elevations in hematocrit and hemoglobin levels throughout follow-up and the cause of these mild elevations is not known. Overall the dogs had no clinical concerns related to these small elevations, ate well and maintained body weight throughout the study.

\section{Limitations of pre-clinical models in general and of the mouse model in particular}

One of the frustrations with pre-clinical studies on vector development has been that the different animal models may yield substantially differing results on the efficacy of 
hepatic gene transfer. While AAV2 transduction has been fairly consistent between species, other serotypes vary considerably. For example, AAV8 transduces murine liver 1-2 logs more efficiently than AAV2 (Fig. 5a) [40]. In the canine model, AAV2, 8, and 9 yield similar results, while these serotypes perform quite distinctly in mouse liver [5, 33, 47]. Interestingly, AAV2 and 8 also directed similar FIX levels in humans $[11,12]$. It is therefore possible that limitations exist in non-murine species that are not yet understood. In the study presented here, FIX levels achieved in hemophilia B dogs, when adjusted for vector dose, were slightly less for AAV2-(Y-F)-M3 (Fig. 2) compared to historical data on AAV2 (Fig. 5b) [30, 31]. However, when considering that the vector constructs are not entirely identical, were purified with different methods, and were not titered side-by-side, and that the animals are an outbred strain, we conclude that AAV2 and AAV2-(Y-F)-M3 capsids perform similarly in dogs. This contrasts with the $0.5-$ to 1 -log difference in expression seen in mouse models $[15,16]$.

Both dogs that were treated with AAV2-(Y-F)-M3 were at the approximately the same age and weight at the time of vector injection (Table 2) but received a threefold different vector dose per $\mathrm{kg}$. During the first 2 months after gene transfer, no clear difference in cFIX expression was obvious. However, the animal treated with the lower vector dose (O19), had a hematoma and required a second round of infusion with normal canine plasma at $\sim 2$ weeks after gene transfer (it takes 8-17 days for aPTT (activity) and 14-27 days for WBTC to return to baseline following transfusion of cFIX protein [46]). During the third month after gene transfer, WBCT and cFIX

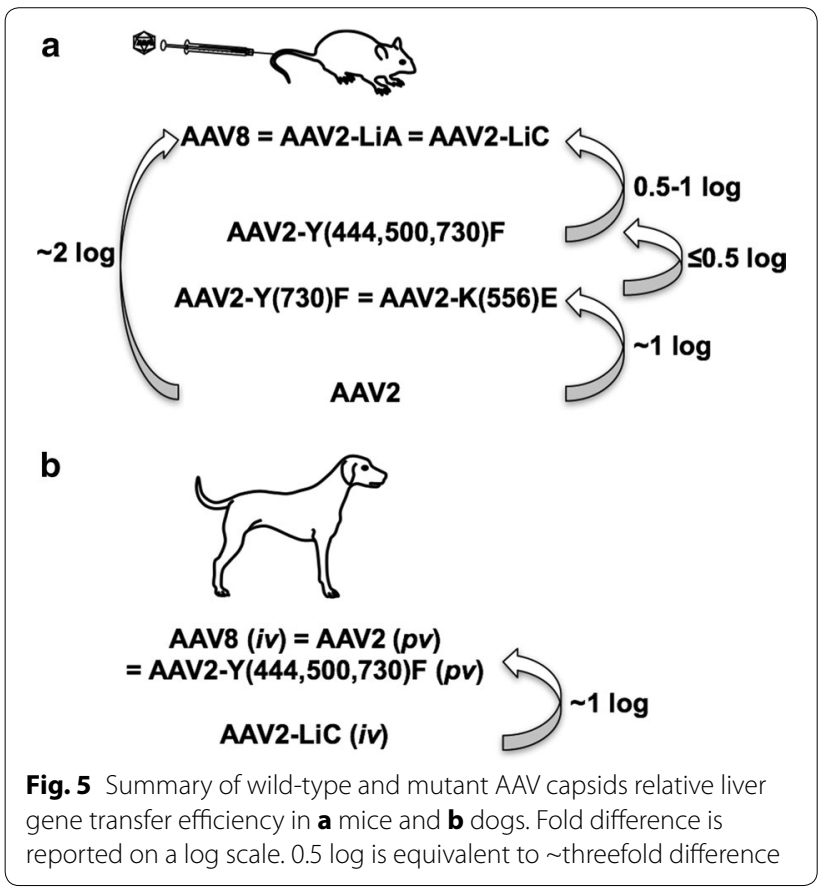

activity measurements suggest an $\sim$ twofold higher levels of expression in the animal receiving a threefold higher dose (O58). Given biological variability, this outcome is within the expected range, while more animals would be required to determine an exact dose response.

In contrast to the introduction of point mutations to eliminate specific $\mathrm{L}, \mathrm{Y}, \mathrm{T}$, and $\mathrm{S}$ residues, AAV2-LiC capsid had been obtained through a molecular evolution strategy, involving generation of a combinatorial capsid library and repeated in vivo selection in murine liver. Liverderived FIX expression after peripheral vein injection of AAV2-LiC into different strains of mice is as high as that obtained with AAV8 (Fig. 5a) [20], resulting in induction of robust immune tolerance to FIX in hemophilia $B$ mice (Fig. 3a-c). However, AAV2-LiC performed poorly in the canine model (Fig. 4), with substantially lower efficacy than for example previously reported for AAV8 (Fig. 5b) $[33,48]$. We therefore conclude that selection for optimal variants in mouse liver likely results in mouse-specific efficacy that does not translate to other species.

\section{Implications for future studies and translation}

In order to better predict performance of hepatic gene transfer with a particular capsid in human patients, two strategies have been adapted: studies in non-human primates and experiments in immune deficient mice whose livers have been partially reconstituted with human hepatocytes $[4,5,35,49,50]$. In the humanized mouse model, we found that different serotypes had highly distinct transduction efficiencies of human hepatocytes, which were often opposite to their relative performance in mouse hepatocytes [5]. An advantage of this model is that human and mouse cell transduction can be directly compared in the same animal. Of the different capsids tested, engineered AAV3 (containing S663V and T492V mutations) outperformed AAV5, 8, and 9 in human hepatocytes [5]. Others who used this model to select for capsids from a "shuffled" library, representing recombined sequences derived from multiple serotypes, also found a capsid primarily consisting of AAV3 sequences to most optimally transduce human hepatocytes [49].

\section{Conclusions}

While only clinical trials can ultimately determine suitability for human gene therapy, it has become clear that results in mouse liver are often not predictive of efficacy in other species or in human liver, so that alternative models need to be used to test rationally designed capsid variants and to select for capsids via directed molecular evolution. New data emerging from ongoing clinical trials evaluating different AAV serotypes will aid in informing which animal model provides the best predictive value for human liver gene transfer efficacy. 


\section{Additional files}

Additional file 1: Table S1. Clinical chemistry panel for hemophilia B dog O19. Baseline values obtained prior to vector. Pre refers to blood collected on the day of vector administrations and post and onward refer to time points after vector delivery. Low values are colored blue, values within normal ranges are black, and values above normal ranges are red.

Additional file 2: Table S2. Clinical chemistry panel for hemophilia $\mathrm{B}$ dog O58. Baseline values obtained prior to vector. Day 0 refers to the day of vector administration. Low values are colored blue, values within normal ranges are black, and values above normal ranges are red.

Additional file 3: Figure S1. No evidence of bethesda inhibitors in either AAV2-(Y-F)-M3 or AAV2-LiC vector treated hemophilia B dogs. Plasma from selected time points was measured for Bethesda inhibitor titer and are reported as Bethesda units per $\mathrm{mL}(\mathrm{BU} / \mathrm{mL})$. Data for each animal and the respective vector, delivery route, and dose are represented by a different color (O19-blue, O58-red, Bruce-Green). AAV2-M3 is an abbreviation of AAV2-(Y-F)-M3. PV portal vein, IV intravenous.

Additional file 4: Table S3. Clinical chemistry panel for hemophilia B dog Bruce. Baseline values obtained prior to vector. Day 0 refers to the day of vector administration. Low values are colored blue, values within normal ranges are black, and values above normal ranges are red.

\section{Abbreviations}

hFIX: human factor IX; F9: factor IX gene; CFIX: canine factor IX; FVIII: factor VIII; AAV: adeno-associated virus; SSAAV: single-stranded AAV; $M H C$ I: major histocompatibility complex class I; HB mice: hemophilia B null-mutation mice; BU: Bethesda unit; ELISA: enzyme-linked immunosorbent assay; aPTT: activated partial thromboplastin time; NABs: neutralizing antibodies; WBCT: whole blood clotting time; ALT: alanine transaminase; CBC: complete blood count.

\section{Authors' contributions}

DMM, TCN, EPM, BP, IZ, and DM performed experiments. DMM, TC, SZ, AS, and RWH designed experiments, analyzed and interpreted data, and wrote the manuscript. DMM and RHW supervised the study. All authors read and approved the final manuscript.

\section{Author details}

${ }^{1}$ Department of Pediatrics, University of Florida, Gainesville, FL 32610, USA.

${ }^{2}$ Department of Pathology and Laboratory Medicine, University of North Carolina-Chapel Hill, Chapel Hill, NC 27599, USA.

\section{Acknowledgements}

Not applicable.

\section{Competing interests}

RWH received royalty payments from Spark Therapeutics for license of AAV gene transfer technology and serves on a scientific advisory board for Applied Genetic Technologies Corporation (AGTC).

\section{Funding}

This research was supported by a National Institutes of Health Grant R01 HL097088 (SZ, AS, RWH) and HL063098 (TCN), Bayer Hemophilia Early Career Investigator Award (DMM), and a Children's Miracle Network Grant-UF Department of Pediatrics Grant (DMM and BP).

\section{Publisher's Note}

Springer Nature remains neutral with regard to jurisdictional claims in published maps and institutional affiliations.

Received: 3 April 2017 Accepted: 25 April 2017

Published online: 01 May 2017
References

1. Kumar SR, Markusic DM, Biswas M, High KA, Herzog RW. Clinical development of gene therapy: results and lessons from recent successes. Mol Ther Methods Clin Dev. 2016;3:16034.

2. Herzog RW. A cure for hemophilia: the promise becomes a reality. Mol Ther. 2016;24:1503-4.

3. Dolgin E. Early clinical data raise the bar for hemophilia gene therapies. Nat Biotechnol. 2016;34:999-1001.

4. Li S, Ling C, Zhong L, Li M, Su Q, He R, Tang Q, Greiner DL, Shultz LD, Brehm $M A$, et al. Efficient and targeted transduction of nonhuman primate liver with systemically delivered optimized AAV3B vectors. Mol Ther. 2015;23:1867-76.

5. Vercauteren K, Hoffman BE, Zolotukhin I, Keeler GD, Xiao JW, BasnerTschakarjan E, High KA, Ertl HC, Rice CM, Srivastava A, et al. Superior in vivo transduction of human hepatocytes using engineered AAV3 capsid. Mol Ther. 2016;24:1042-9.

6. Ertl HCJ, High KA. Impact of AAV capsid-specific T-cell responses on design and outcome of clinical gene transfer trials with recombinant adeno-associated viral vectors: An evolving controversy. Hum Gene Ther. 2017;28(4):328-37.

7. Pien GC, Basner-Tschakarjan E, Hui DJ, Mentlik AN, Finn JD, Hasbrouck NC, Zhou S, Murphy SL, Maus MV, Mingozzi F, et al. Capsid antigen presentation flags human hepatocytes for destruction after transduction by adeno-associated viral vectors. J Clin Invest. 2009;119:1688-95.

8. Li C, He Y, Nicolson S, Hirsch M, Weinberg MS, Zhang P, Kafri T, Samulski RJ. Adeno-associated virus capsid antigen presentation is dependent on endosomal escape. J Clin Invest. 2013;123:1390-401.

9. Martino AT, Basner-Tschakarjan E, Markusic DM, Finn JD, Hinderer C, Zhou S, Ostrov DA, Srivastava A, Ertl HC, Terhorst C, et al. Engineered AAV vector minimizes in vivo targeting of transduced hepatocytes by capsid-specific CD8+ T cells. Blood. 2013;121:2224-33.

10. Mingozzi F, Maus MV, Hui DJ, Sabatino DE, Murphy SL, Rasko JE, Ragni MV, Manno CS, Sommer J, Jiang H, et al. CD8(+) T-cell responses to adenoassociated virus capsid in humans. Nat Med. 2007;13:419-22.

11. Manno CS, Pierce GF, Arruda VR, Glader B, Ragni M, Rasko JJ, Ozelo MC, Hoots K, Blatt P, Konkle B, et al. Successful transduction of liver in hemophilia by AAV-factor IX and limitations imposed by the host immune response. Nat Med. 2006;12:342-7.

12. Nathwani AC, Tuddenham EG, Rangarajan S, Rosales C, Mclntosh J, Linch DC, Chowdary P, Riddell A, Pie AJ, Harrington C, et al. Adenovirus-associated virus vector-mediated gene transfer in hemophilia B. N Engl J Med. 2011;365:2357-65.

13. Nathwani AC, Reiss UM, Tuddenham EG, Rosales C, Chowdary P, McIntosh J, Della Peruta M, Lheriteau E, Patel N, Raj D, et al. Long-term safety and efficacy of factor IX gene therapy in hemophilia B. N Engl J Med. 2014;371:1994-2004.

14. Zhong L, Zhao W, Wu J, Li B, Zolotukhin S, Govindasamy L, AgbandjeMcKenna M, Srivastava A. A dual role of EGFR protein tyrosine kinase signaling in ubiquitination of AAV2 capsids and viral second-strand DNA synthesis. Mol Ther. 2007;15:1323-30.

15. Zhong L, Li B, Mah CS, Govindasamy L, Agbandje-McKenna M, Cooper M, Herzog RW, Zolotukhin I, Warrington KH Jr, Weigel-Van Aken KA, et al. Next generation of adeno-associated virus 2 vectors: point mutations in tyrosines lead to high-efficiency transduction at lower doses. Proc Natl Acad Sci USA. 2008;105:7827-32.

16. Markusic DM, Herzog RW, Aslanidi GV, Hoffman BE, Li B, Li M, Jayandharan GR, Ling C, Zolotukhin I, Ma W, et al. High-efficiency transduction and correction of murine hemophilia B using AAV2 vectors devoid of multiple surface-exposed tyrosines. Mol Ther. 2010;18:2048-56.

17. Srivastava A. Adeno-associated virus: the naturally occurring virus versus the recombinant vector. Hum Gene Ther. 2016;27:1-6.

18. Aslanidi GV, Rivers AE, Ortiz L, Song L, Ling C, Govindasamy L, Van Vliet K, Tan M, Agbandje-McKenna M, Srivastava A. Optimization of the capsid of recombinant adeno-associated virus 2 (AAV2) vectors: the final threshold? PLoS ONE. 2013;8:e59142.

19. Li B, Ma W, Ling C, Van Vliet K, Huang LY, Agbandje-McKenna M, Srivastava A, Aslanidi GV. Site-directed mutagenesis of surface-exposed lysine residues leads to improved transduction by AAV2, but not AAV8, vectors in murine hepatocytes in vivo. Hum Gene Ther Methods. 2015;26:211-20. 
20. Marsic D, Govindasamy L, Currlin S, Markusic DM, Tseng YS, Herzog RW, Agbandje-McKenna M, Zolotukhin S. Vector design Tour de Force: integrating combinatorial and rational approaches to derive novel adenoassociated virus variants. Mol Ther. 2014;22:1900-9.

21. Grimm D, Zolotukhin S. E pluribus unum: 50 years of research, millions of viruses, and one goal-tailored acceleration of AAV evolution. Mol Ther. 2015;23:1819-31.

22. Mingozzi F, Liu YL, Dobrzynski E, Kaufhold A, Liu JH, Wang Y, Arruda VR, High KA, Herzog RW. Induction of immune tolerance to coagulation factor IX antigen by in vivo hepatic gene transfer. J Clin Invest. 2003;111:1347-56.

23. Sarkar D, Biswas M, Liao G, Seay HR, Perrin GQ, Markusic DM, Hoffman BE, Brusko TM, Terhorst C, Herzog RW. Ex vivo expanded autologous polyclonal regulatory $T$ cells suppress inhibitor formation in hemophilia. Mol Ther Methods Clin Dev. 2014;1:14030.

24. Sherman A, Schlachterman A, Cooper M, Merricks EP, Raymer RA, Bellinger DA, Herzog RW, Nichols TC. Portal vein delivery of viral vectors for gene therapy for hemophilia. Methods Mol Biol. 2014;1114:413-26.

25. Markusic DM, Hoffman BE, Perrin GQ, Nayak S, Wang X, LoDuca PA, High KA, Herzog RW. Effective gene therapy for haemophilic mice with pathogenic factor IX antibodies. EMBO Mol Med. 2013;5:1698-709.

26. Herzog RW, Mount JD, Arruda VR, High KA, Lothrop CD Jr. Muscledirected gene transfer and transient immune suppression result in sustained partial correction of canine hemophilia B caused by a null mutation. Mol Ther. 2001;4:192-200.

27. Gabriel N, Hareendran S, Sen D, Gadkari RA, Sudha G, Selot R, Hussain M, Dhaksnamoorthy R, Samuel R, Srinivasan N, et al. Bioengineering of AAV2 capsid at specific serine, threonine, or lysine residues improves its transduction efficiency in vitro and in vivo. Hum Gene Ther Methods. 2013;24:80-93.

28. Sabatino DE, Nichols TC, Merricks E, Bellinger DA, Herzog RW, Monahan PE. Animal models of hemophilia. Prog Mol Biol Transl Sci. 2012;105:151-209.

29. Nichols TC, Whitford MH, Arruda VR, Stedman HH, Kay MA, High KA. Translational data from adeno-associated virus-mediated gene therapy of hemophilia B in dogs. Hum Gene Ther Clin Dev. 2015;26:5-14.

30. Mount JD, Herzog RW, Tillson DM, Goodman SA, Robinson N, McCleland ML, Bellinger D, Nichols TC, Arruda VR, Lothrop CD Jr, High KA. Sustained phenotypic correction of hemophilia B dogs with a factor IX null mutation by liver-directed gene therapy. Blood. 2002;99:2670-6.

31. Niemeyer GP, Herzog RW, Mount J, Arruda VR, Tillson DM, Hathcock J, van Ginkel FW, High KA, Lothrop CD Jr. Long-term correction of inhibitorprone hemophilia B dogs treated with liver-directed AAV2-mediated factor IX gene therapy. Blood. 2009;113:797-806.

32. Nichols TC, Hough C, Agerso H, Ezban M, Lillicrap D. Canine models of inherited bleeding disorders in the development of coagulation assays, novel protein replacement and gene therapies. J Thromb Haemost. 2016;14:894-905.

33. Crudele JM, Finn JD, Siner Jl, Martin NB, Niemeyer GP, Zhou S, Mingozzi F, Lothrop CD Jr, Arruda VR. AAV liver expression of FIX-Padua prevents and eradicates FIX inhibitor without increasing thrombogenicity in hemophilia B dogs and mice. Blood. 2015;125:1553-61.

34. Markusic DM, Herzog RW. Liver-directed adeno-associated viral gene therapy for hemophilia. J Genet Syndr Gene Ther. 2012;1:1-9.

35. Ling C, Yin Z, Li J, Zhang D, Aslanidi G, Srivastava A. Strategies to generate high-titer, high-potency recombinant AAV3 serotype vectors. Mol Ther Methods Clin Dev. 2016:3:16029.
36. Ling C, Li B, Ma W, Srivastava A. Development of optimized AAV serotype vectors for high-efficiency transduction at further reduced doses. Hum Gene Ther Methods. 2016;27:143-9.

37. Ling C, Wang Y, Zhang Y, Ejjigani A, Yin Z, Lu Y, Wang L, Wang M, Li J, Hu Z, et al. Selective in vivo targeting of human liver tumors by optimized AAV3 vectors in a murine xenograft model. Hum Gene Ther. 2014;25:1023-34.

38. Leithe $E$, Rivedal E. Epidermal growth factor regulates ubiquitination, internalization and proteasome-dependent degradation of connexin43. J Cell Sci. 2004;117:1211-20.

39. Nakai H, Fuess S, Storm TA, Muramatsu S, Nara Y, Kay MA. Unrestricted hepatocyte transduction with adeno-associated virus serotype 8 vectors in mice. J Virol. 2005;79:214-24.

40. Cooper M, Nayak S, Hoffman BE, Terhorst C, Cao O, Herzog RW. Improved induction of immune tolerance to factor IX by hepatic AAV-8 gene transfer. Hum Gene Ther. 2009;20:767-76.

41. Nakai H, Thomas CE, Storm TA, Fuess S, Powell S, Wright JF, Kay MA. A limited number of transducible hepatocytes restricts a wide-range linear vector dose response in recombinant adeno-associated virus-mediated liver transduction. J Virol. 2002;76:11343-9.

42. Zhao W, Zhong L, Wu J, Chen L, Qing K, Weigel-Kelley KA, Larsen SH, Shou W, Warrington KH Jr, Srivastava A. Role of cellular FKBP52 protein in intracellular trafficking of recombinant adeno-associated virus 2 vectors. Virology. 2006;353:283-93.

43. Zhong L, Li W, Yang Z, Chen L, Li Y, Qing K, Weigel-Kelley KA, Yoder MC, Shou W, Srivastava A. Improved transduction of primary murine hepatocytes by recombinant adeno-associated virus 2 vectors in vivo. Gene Ther. 2004;11:1165-9.

44. Finn JD, Hui D, Downey HD, Dunn D, Pien GC, Mingozzi F, Zhou S, High KA. Proteasome inhibitors decrease AAV2 capsid derived peptide epitope presentation on MHC class I following transduction. Mol Ther. 2010;18:135-42.

45. Monahan PE, Lothrop CD, Sun J, Hirsch ML, Kafri T, Kantor B, Sarkar R, Tillson DM, Elia JR, Samulski RJ. Proteasome inhibitors enhance gene delivery by AAV virus vectors expressing large genomes in hemophilia mouse and dog models: a strategy for broad clinical application. Mol Ther. 2010;18:1907-16.

46. Nichols TC, Franck HW, Franck CT, De Friess N, Raymer RA, Merricks EP. Sensitivity of whole blood clotting time and activated partial thromboplastin time for factor IX: relevance to gene therapy and determination of post-transfusion elimination time of canine factor IX in hemophilia B dogs. J Thromb Haemost. 2012;10:474-6.

47. Sabatino DE, Lange AM, Altynova ES, Sarkar R, Zhou S, Merricks EP, Franck HG, Nichols TC, Arruda VR, Kazazian HH Jr. Efficacy and safety of long-term prophylaxis in severe hemophilia A dogs following liver gene therapy using AAV vectors. Mol Ther. 2011;19:442-9.

48. Wang L, Calcedo R, Nichols TC, Bellinger DA, Dillow A, Verma IM, Wilson $J M$. Sustained correction of disease in naive and AAV2-pretreated hemophilia B dogs: AAV2/8-mediated, liver-directed gene therapy. Blood. 2005:105:3079-86.

49. Lisowski L, Dane AP, Chu K, Zhang Y, Cunningham SC, Wilson EM, Nygaard S, Grompe M, Alexander IE, Kay MA. Selection and evaluation of clinically relevant AAV variants in a xenograft liver model. Nature. 2014;506:382-6.

50. Bissig-Choisat B, Wang L, Legras X, Saha PK, Chen L, Bell P, Pankowicz FP, Hill MC, Barzi M, Kettlun Leyton C, et al. Development and rescue of human familial hypercholesterolaemia in a xenograft mouse model. Nat Commun. 2015;6:7339. 